\title{
Multifactorial treatment increases endothelial progenitor cells in patients with type 2 diabetes
}

\author{
H. Reinhard • P. Karl Jacobsen • M. Lajer • \\ N. Pedersen • N. Billestrup • T. Mandrup-Poulsen • \\ H.-H. Parving $\cdot$ P. Rossing
}

Received: 18 March 2010 /Accepted: 11 June 2010/Published online: 6 July 2010

(C) Springer-Verlag 2010

\begin{abstract}
Aims/hypothesis Endothelial progenitor cells (EPC) augment vascular repair and neovascularisation. Patients with type 2 diabetes have reduced EPC and increased risk of cardiovascular disease (CVD), which is reduced by multifactorial intervention. Our aim, therefore, was to evaluate in
\end{abstract}

H. Reinhard $(\bowtie) \cdot$ P. K. Jacobsen $\cdot$ M. Lajer $\cdot$ N. Pedersen $\cdot$

P. Rossing

Steno Diabetes Center,

Niels Steensenvej 1,

DK-2820 Gentofte, Denmark

e-mail: hnri@steno.dk

P. K. Jacobsen

The Heart Centre,

Rigshospitalet,

Copenhagen, Denmark

N. Billestrup · T. Mandrup-Poulsen

Department of Translational Diabetology,

Hagedorn Research Institute,

Gentofte, Denmark

T. Mandrup-Poulsen

Core Unit for Medical Research Methodology,

Department of Biomedical Sciences, University of Copenhagen,

Copenhagen, Denmark

T. Mandrup-Poulsen

Department of Medicine and Surgery, Karolinska Institutet,

Stockholm, Sweden

H.-H. Parving

Department of Medical Endocrinology, Rigshospitalet,

University Hospital of Copenhagen,

Copenhagen, Denmark

H.-H. Parving

Faculty of Health Science, Aarhus University,

Aarhus, Denmark type 2 diabetic patients whether the numbers of EPC derived from peripheral blood mononuclear cells is influenced by a multifactorial treatment strategy.

Methods We enrolled 28 patients newly referred for initiation of multifactorial treatment, which consisted of improving glycaemic, lipid and blood pressure control, as well as antithrombotic therapy and lifestyle modification. EPC count was assessed by in vitro cultures at baseline and after 90 days of treatment. After 7 days in culture, we identified EPC by fluorescent staining of attached cells. Patients were treated with metformin, aspirin, statins and angiotensin II receptor blockers, and divided accordingly into groups of mono-, dual-, triple- or quadruple therapy.

Results After 90 days of treatment, glycaemic control improved and total cholesterol decreased. Multifactorial intervention for 90 days significantly increased EPC count in cultures by $35 \%$ (from 105 [SE 8] to 140 [11] cells per field $[p=0.002])$. The change in EPC among patients with quadruple therapy was higher $(63 \%)$ than in untreated patients $(-32 \%, p=0.043)$.

Conclusions/interpretation Numbers of EPC derived from peripheral blood mononuclear cells increased significantly after multifactorial intervention in type 2 diabetic patients. It remains to be shown whether these changes contribute to the beneficial effects of multifactorial intervention on diabetic micro- and macroangiopathy.

Keywords Endothelial progenitor cells . Multifactorial cardiovascular intervention. Type 2 diabetes mellitus
Abbreviations
CVD Cardiovascular disease
EPC Endothelial progenitor cells
UKPDS UK Prospective Diabetes Study 


\section{Introduction}

Cardiovascular disease (CVD) is a major determinant of increased morbidity and mortality rates in type 2 diabetic patients. Current treatment strategies for CVD focus on reducing established risk factors. Few studies have explored the potential of promoting vascular repair, regeneration or even neovascularisation.

Endothelial progenitor cells (EPC) are involved in adult neovascularisation and repair of damaged endothelium, important for maintenance of vascular integrity. Evidence is emerging that EPC number (count) and function are altered in clinical conditions characterised by high risk of CVD. Decreased EPC count and function are seen in patients with type 2 diabetes [1]. Furthermore, circulating $\mathrm{CD} 34^{+}$cells independently predicted CVD and death in patients with metabolic syndrome [2]. EPC are increased in non-diabetic patients treated with simvastatin [3] or ACEinhibitors [4], thus mobilisation may improve CVD prognosis.

Morbidity and mortality rates from small and large vessel lesions in microalbuminuric type 2 diabetic patients are reduced by multifactorial intervention. Despite the established relationship between cardiovascular risk factors and EPC count, little is known about the influence of treatments of type 2 diabetes patients on EPC count. We therefore aimed to evaluate whether the numbers of early $\mathrm{EPC}$, in the form of cells derived from peripheral blood mononuclear cells and cultured under endothelial cells conditions, are influenced by multifactorial treatment in type 2 diabetic patients.

\section{Methods}

Participants and study procedures We enrolled 33 type 2 diabetic patients consecutively referred to the outpatient clinic at Steno Diabetes Center for initiation of multifactorial treatment designed to reduce the risk of micro- and macrovascular complications. None of the patients had been previously diagnosed or medically treated for CVD with the exception of glucose-lowering treatment (received by 14 patients at baseline). At baseline, all patients were encouraged to start multifactorial treatment including improvement of glycaemic control, lipidlowering intervention, antihypertensive and antithrombotic therapy, and lifestyle modification according to international guidelines [5]. Patients were treated with metformin, aspirin, statins and angiotensin II receptor blockers, and subsequently subdivided into groups of mono-, dual-, triple- or quadruple therapy. The lifestyle programme consisted of dietary advice, exercise, smoking cessation and weight loss.
Of the 33 patients, five were excluded: two who were diagnosed with maturity-onset diabetes of the young; one who had a urinary tract infection at the beginning of the study; and two who were taking antimetabolites due to eczema and a postoperative breast cancer. The 28 patients completing the study were considered free from diseases known to mobilise EPC.

Non-fasting blood samples were obtained after $10 \mathrm{~min}$ of rest and analysed according to standard procedures. The 10 year UK Prospective Diabetes Study (UKPDS) $\mathrm{CHD} /$ stroke risk engine scores were calculated in all patients at baseline and after 30 and 90 days [6].

The study protocol was approved by the local Ethical Committee and all participants gave informed consent.

EPC isolation and in vitro culture Human mononuclear cells were isolated from peripheral blood at baseline, and after 30 and 90 days, using density gradient centrifugation (Ficoll Lymphoprep; Axis Shield, Oslo, Norway). Mononuclear cells $\left(1 \times 10^{7}\right)$ in $3 \mathrm{ml}$ of endothelial basal medium (Lonza, Basel, Switzerland) were seeded in six-well plates coated with human fibronectin (BD BioCoat; BD Biosciences, Mississauga, ON, Canada) [3, 4, 7-10]. After 4 days of culture, non-adherent cells were removed and culturing continued for three additional days. Early EPC were identified by fluorescent chemical detection of attached cells using $1 \mathrm{mg} / \mathrm{ml}$ acetylated LDL-Dil uptake (Invitrogen, Carlsbad, CA, USA). Adherent cells were fixed and stained with $10 \mu \mathrm{g} / \mathrm{ml} \mathrm{FITC-labelled} \mathrm{Ulex} \mathrm{europaeus}$ agglutinin 1 (Sigma Aldrich, St Louis, MO, USA). Doublestained EPC were counted in ten random fields by using an inverted fluorescence microscope and Image-pro software (Media Cybernetic, Bethesda, MD, USA). To facilitate counting and improve reproducibility, all cultures were nuclear stained with DAPI. EPC counting was verified by two independent investigators blinded to clinical data, treatment of the patient and visit number. The EPC count at baseline and during follow-up was successfully measured in 26 patients. An inter-observer variability of $<2 \%$ and day-to-day variation of $<11 \%$ were observed.

Statistical analysis Data were expressed as means $\pm \mathrm{SD} / \mathrm{SE}$ or medians (range). Comparisons between groups were performed by the unpaired or paired Student's $t$ test, or Spearman's rho as described. A $p$ value of $p<0.05$ was considered to be statistically significant.

\section{Results}

Baseline characteristics and the effect of multifactorial intervention during 30 or 90 days are shown in Table 1. 
Table 1 Baseline clinical characteristics and effect of multifactorial intervention during 30 or 90 days

\begin{tabular}{|c|c|c|c|c|c|}
\hline Variables & Baseline & 30days & 90days & $p$ value $^{\mathrm{a}}$ & $p$ value $^{\mathrm{b}}$ \\
\hline$n$ & 28 & 28 & 28 & - & - \\
\hline Age (years) & $51 \pm 2$ & - & - & - & - \\
\hline Known diabetes duration (years) & $3(0-15)$ & - & - & - & - \\
\hline $\mathrm{HbA}_{1 \mathrm{c}}(\%)$ & $7.5 \pm 0.2$ & $7.1 \pm 0.2$ & $6.9 \pm 0.2$ & 0.02 & 0.009 \\
\hline Plasma cholesterol (mmol/l) & $4.9 \pm 0.1$ & $3.6 \pm 0.2$ & $3.6 \pm 0.2$ & $<0.001$ & $<0.001$ \\
\hline BMI $\left(\mathrm{kg} / \mathrm{m}^{2}\right)$ & $32.0 \pm 1.1$ & $30.9 \pm 1.6$ & $31.4 \pm 1.1$ & 0.27 & 0.04 \\
\hline High-sensitivity CRP (mg/l) & $9.7 \pm 1.4$ & $10.9 \pm 1.4$ & $11.0 \pm 0.8$ & 0.13 & 0.96 \\
\hline Systolic BP (mmHg) & $139 \pm 3$ & $132 \pm 6$ & $135 \pm 3$ & 0.18 & 0.11 \\
\hline Diastolic BP (mmHg) & $89 \pm 2$ & $84 \pm 4$ & $87 \pm 2$ & 0.15 & 0.31 \\
\hline Urinary AER (mg/24 h) & $22(3-508)$ & $22(6-2419)$ & $18(5-731)$ & 0.92 & 0.79 \\
\hline Therapy combinations ${ }^{\mathrm{c}}$ & & & & - & - \\
\hline Mono $(n)$ & 4 & 4 & 4 & - & - \\
\hline Dual $(n)$ & 3 & 3 & 3 & - & - \\
\hline Triple $(n)$ & 12 & 12 & 12 & - & - \\
\hline Quadruple $(n)$ & 5 & 5 & 5 & - & - \\
\hline Medication & & & & - & - \\
\hline Simvastatin $(n)$ & 23 & 23 & 23 & - & - \\
\hline Aspirin $(n)$ & 20 & 20 & 20 & - & - \\
\hline $\mathrm{ARB}(n)$ & 7 & 7 & 7 & - & - \\
\hline Metformin $(n)$ & 21 & 21 & 21 & - & - \\
\hline Metformin dose ${ }^{\mathrm{d}}(\mathrm{mg})$ & $1000(500-2000)$ & $1500(500-2000)$ & $2000(500-3000)$ & - & - \\
\hline UKPDS CHD $(\%)^{\mathrm{e}}$ & $21.4 \pm 3.0$ & $14.1 \pm 2.1$ & $12.9 \pm 2.3$ & $<0.001$ & $<0.001$ \\
\hline UKPDS fatal CHD $(\%)^{\mathrm{e}}$ & $14.4 \pm 2.5$ & $9.2 \pm 1.7$ & $8.5 \pm 2.0$ & 0.001 & 0.003 \\
\hline UKPDS STROKE $(\%)^{\mathrm{f}}$ & $9.8 \pm 2.2$ & $8.5 \pm 1.9$ & $7.8 \pm 1.9$ & 0.005 & 0.006 \\
\hline UKPDS fatal STROKE $(\%)^{\mathrm{f}}$ & $1.5 \pm 0.3$ & $1.3 \pm 0.3$ & $1.1 \pm 0.3$ & 0.13 & 0.038 \\
\hline Retinopathy $(n)$ & 4 & 4 & 4 & - & - \\
\hline Microalbuminuria $(n)$ & 7 & 7 & 7 & - & - \\
\hline Smoking $(n)$ & 10 & 10 & 10 & - & - \\
\hline
\end{tabular}

Data are mean $\pm \mathrm{SE}$ or median (range)

${ }^{\mathrm{a}}$ Baseline to 30 days; ${ }^{\mathrm{b}}$ baseline to 90 days

${ }^{\mathrm{c}}$ Includes the use of metformin, aspirin, statins and ARB

${ }^{\mathrm{d}}$ Prior to start of the study, 14 patients were taking metformin (median dose 1,000 mg, range 500-1500); one patient had started insulin in addition to metformin treatment

${ }^{\mathrm{e}} 10$ year risk of (non)-fatal $\mathrm{CHD} ;{ }^{\mathrm{f}} 10$ year risk of (non)-fatal stroke

$\mathrm{ARB}$, angiotensin II receptor blocker; CRP, C-reactive protein

EPC count The mean in vitro EPC count at baseline was 105 (SD 42) cells. None of the conventional risk factors, metabolic control or UKPDS risk scores was associated with baseline EPC level ( $p>0.05)$.

After 30 days, the EPC count increased by $15 \%$ from 105 (SE 8) to 124 [9] cells $(p=0.09)$, while 90 days of multifactorial intervention significantly increased the EPC count by $35 \%$ to $140(11, p=0.002)$. The EPC count change was reduced $(-32 \%)$ in medically untreated $(n=2)$ and increased by $63 \%$ in patients with quadruple therapy $(n=5$, $p=0.043$ ). In a scatter plot correlation and when analysed with Spearman's rho, the EPC-increasing effect of treat- ment paralleled the increase in number of drugs used, albeit not significantly ( $p=0.121$, Fig. 1$)$. The use of each drug individually or the changes in UKPDS risk scores were not associated with EPC count $(p>0.05)$.

\section{Discussion}

We have shown that multifactorial treatment aiming to decrease conventional CVD risk factors in type 2 diabetic patients increases the number of in vitroassessed EPC by $35 \%$ after 90 days. The study was 


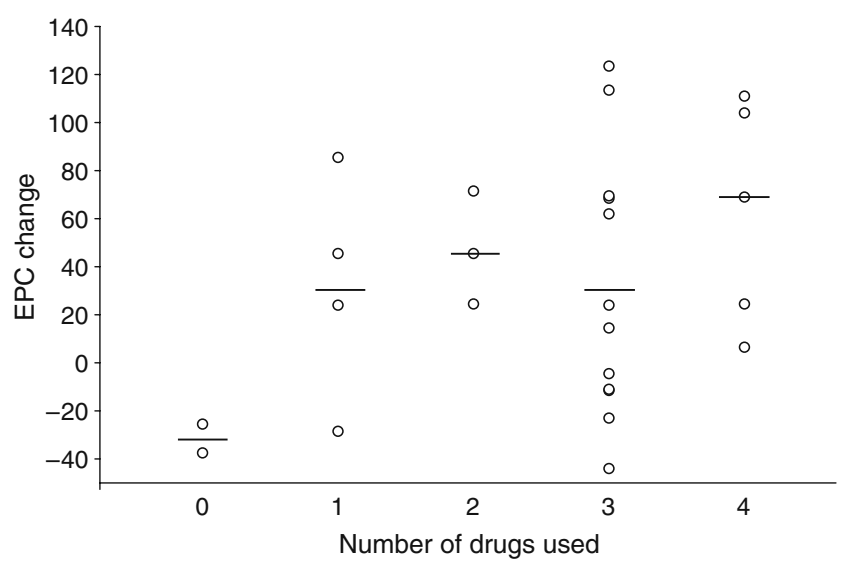

Fig. 1 Scatter plot correlation showing early EPC count after 90 days of treatment according to the number of drugs used in patient with type 2 diabetes. Horizontal bars, median EPC change per group, $p=0.121$

uncontrolled, but the EPC count change was lower in patients not medically treated and increased by $63 \%$ in patients with quadruple therapy. Finally, 90 days of treatment also improved risk scores for cardiovascular outcomes in these patients.

Quadruple therapy consisted of metformin, aspirin, statins and angiotensin II blockers. Statins have previously been shown to mobilise EPC. Whether the mobilising effect of statins is mediated through a stimulating effect on bone marrow, a direct effect on EPC or a combination is not known. However, a direct effect on EPC differentiation in vitro was suggested in a mouse model, using culture conditions similar to ours [9]. Angiotensin II blockers also increase EPC in type 2 diabetes patients [7], the magnitude being comparable to that achieved with statins and independent of blood pressure. The additional EPC count increase associated with quadruple therapy may reflect angiotensin II receptor blockade, as this was used as third or fourth drug of choice. In addition, low-dose aspirin has been shown to increase the EPC count [10]. The question of whether metformin had additional effects on EPC count independently of the glucose-lowering action related to its potential therapeutic benefit in CVD remains to be answered. We were not able to demonstrate any association between EPC count and traditional risk factors or UKPDS risk scores. However, a direct independent medical effect on EPC would mask these relations. Flow cytometry and cell cultures are used to define EPC. The latter method is generally accepted, but methodological inconsistencies exist. In particular, the duration of culturing varies between studies, leading to EPC being defined as either early or late EPC [11]. Differences among these cell types have been described, and although both types of EPC have shown comparable vasculogenic capacity [12], early EPC may not represent true EPC. Acetylated LDL uptake and lectin binding have been used in a number of important EPC studies [3, 4, 710] and constitute a necessary step in measuring cultured EPC count [11]. Additional EPC markers are now recommended, but no consensus on this exists [11]. To allow computerised and unbiased counting, we used DAPI in addition to acetylated LDL uptake and lectin binding. Another point is that cultured EPC count does not in itself correspond to in vivo EPC as determined by flow cytometry. Cultured EPC count merely reflects the EPC present in the blood sample and their function regarding proliferation, survival and successful adhesion. Confirmation of our finding by flow cytometry would have strengthened our study.

Finally, the statistical power to address associations between changes in EPC count and other risk factors or treatment modalities was relatively low due to the small sample size. Our analysis should therefore be interpreted with caution. Our study did not include a control group, because a delay in initiation of multifactorial treatment to prevent CVD was regarded as unethical. However, two patients rejected medical treatment and hence could be studied as controls. We showed an increasing effect with time and a relationship between increase in number of drugs prescribed and EPC count increase after 90 days. However, subgrouping in a relatively small study should be interpreted with caution, even though it was necessary due to the different treatment strategies among the study patients. Furthermore, a seasonal effect cannot be ruled out. The EPC count increase, however, was not associated with time (summer/winter) of inclusion and follow-up. Similarly, EPC count did not change with time among healthy controls (data not shown).

Multifactorial treatment has previously been documented to reduce long-term mortality and CVD morbidity rates by $50 \%$ in type 2 diabetic patients. The present $35 \%$ increase in EPC count nearly corresponds to a correction of the reported $48 \%$ reduction in EPC count demonstrated by similar in vitro culture in type 2 diabetic patients compared with non-diabetic participants [1]. It is not known whether changes in EPC count contribute to risk reduction. However, reduced circulating CD $34^{+}$cells independently predicted cardiovascular events and death in patients with metabolic syndrome (2).

In conclusion, our data demonstrate that the number EPC derived from peripheral blood mononuclear cells increased significantly during multifactorial intervention in type 2 diabetic patients. Whether these changes contribute to the beneficial effects of multifactorial intervention on CVD in such patients remains to be shown. 
Acknowledgements The authors wish to acknowledge the work of lab technicians U. M. Smidt, L. Pietraszeck and A. G. Lundgaard, who are employees at Steno Diabetes Center. The study was supported by The Danish Diabetes Association and Steno Diabetes Center.

Duality of interest The authors declare that there is no duality of interest associated with this manuscript.

\section{References}

1. Tepper OM, Galiano RD, Capla JM et al (2002) Human endothelial progenitor cells from type II diabetics exhibit impaired proliferation, adhesion, and incorporation into vascular structures. Circulation 106:2781-2786

2. Fadini GP, de Kreutzenberg S, Agostini C et al (2009) Low CD $34^{+}$cell count and metabolic syndrome synergistically increase the risk of adverse outcomes. Atherosclerosis 207:213-219

3. Vasa M, Fichtlscherer S, Adler K et al (2001) Increase in circulating endothelial progenitor cells by statin therapy in patients with stable coronary artery disease. Circulation 103:2885-2890

4. Min TQ, Zhu CJ, Xiang WX, Hui ZJ, Peng SY (2004) Improvement in endothelial progenitor cells from peripheral blood by ramipril therapy in patients with stable coronary artery disease. Cardiovasc Drugs Ther 18:203-209
5. American Diabetes Association (2009) Standards of medical care in diabetes-2009. Diab Care 32:S13-S61

6. Stevens RJ, Kothari V, Adler AI, Stratton IM (2001) The UKPDS risk engine: a model for the risk of coronary heart disease in type II diabetes (UKPDS 56). Clin Sci (Lond) 101:671-679

7. Bahlmann FH, de Groot K, Mueller O, Hertel B, Haller H, Fliser D (2005) Stimulation of endothelial progenitor cells: a new putative therapeutic effect of angiotensin II receptor antagonists. Hypertension 45:526-529

8. Vasa M, Fichtlscherer S, Aicher A et al (2001) Number and migratory activity of circulating endothelial progenitor cells inversely correlate with risk factors for coronary artery disease. Circ Res 89:E1-E7

9. Dimmeler S, Aicher A, Vasa M et al (2001) HMG-CoA reductase inhibitors (statins) increase endothelial progenitor cells via the PI 3-kinase/Akt pathway. J Clin Invest 108:391-397

10. Hu Z, Zhang F, Yang $Z$ et al (2008) Low-dose aspirin promotes endothelial progenitor cell migration and adhesion and prevents senescence. Cell Biol Int 32:761-768

11. Fadini GP, Baesso I, Albiero M, Sartore S, Agostini C, Avogaro A (2008) Technical notes on endothelial progenitor cells: ways to escape from the knowledge plateau. Atherosclerosis 197:496-503

12. Hur J, Yoon CH, Kim HS et al (2004) Characterization of two types of endothelial progenitor cells and their different contributions to neovasculogenesis. Arterioscler Thromb Vasc Biol 24:288-293 